\title{
Emerging Biomarkers of Alcohol Consumption: Clinical and Forensic Applications
}

\author{
Vincenza Bianchi ${ }^{1, *}$, Alessia Raspagni ${ }^{2}$ and Carlo Arfini ${ }^{1}$ \\ ${ }^{1}$ Toxicology Reference Laboratory, SS Antonio e Biagio e C Arrigo Hospital, Alessandria, Italy \\ ${ }^{2}$ School of Medicine, Insubria University, Varese, Italy
}

\begin{abstract}
Alcohol-related problems are typically associated with medical, economic and social issues.
The reduction of the problem can only occur if effective strategies to prevent, diagnose and treat alcohol abuse are developed.

Real help could come from laboratory tests that represent objective evidence of alcohol problems.

Authors present new biomarkers as Carbohydrate-deficient transferrin (CDT), Ethyl Glucuronide and Ethyl Sulfate ( EtG and EtS)), Fatty Acids Ethyl Esters (FAEE) anf Phosphatidy ethanol (PEth) in differents biological samples.
\end{abstract}

Keywords: Alcohol abuse biomarkers, CDT, EtG, PEth, FAEE.

\section{INTRODUCTION}

The consumption of alcohol, of which European countries have the dubious distinction, is growing strongly and with it all the health issues related to it [1]. About a quarter of treatment costs for the population, in particular for hospitalization, is related to excessive alcohol consumption in someway or other [2] and despite the high standard of living of European nations, the excessive consumption of alcohol may lead to lower life expectancy [3].

The document "Alcohol in Europe: a public health perspective" [4] reports that the average consumption per capita of a European is 11 litres per annum, compared to 7 litres of Americans.

In the countries of Eastern Europe (Hungary, Romania and the Czech Republic) it is observed a higher consumption of alcohol is observed (>17 L pa).

More and more young people are addicted to alcohol: kids of 11 years admit they drink and, on average, by 14 they have experienced their first intoxication.

The 2003 Eurobarometer survey shows that while it is true that men drink more than women, the percentage of women who drink is increasing, and even during pregnancy $25-50 \%$ say they do not quit. The non-drinkers or those classified as abstainers, however, are gradually declining. It is estimated that in Europe there are 23 million alcoholdependent people.

*Address correspondence to this author at the Toxicology Reference Laboratory. SS Antonio e Biagio e C Arrigo Hospital, Alessandria, Italy; Tel: 0131 206214; Fax: 0131 206075;

E-mail: vbianchi@ospedale.al.it
Regarding the type of drinks, beer accounts for $44 \%$ of alcoholic beverages, wine $34 \%$ and spirits $23 \%$. Beer is used mainly in Northern Europe, while wine is typical of Southern Europe.

Although there is not a clear threshold to define a heavy drinker, epidemiological data indicates that doses greater than $300 \mathrm{~g} /$ week for man and $200 \mathrm{~g} /$ week for the woman are significantly hazardous to health. Clinically, the hazardous alcohol intake abuse is different from alcohol abuse because when people abuse in alcohol health is at risk and they show social problems and skills.

In fact alcoholism is a very serious set of problems involving addiction, increased tolerance and withdrawal symptoms when not drinking.

Alcohol-related problems are typically associated with medical, economic and social issues. Over-consumption is the cause of premature death, but the excessive use of alcohol is also implicated in criminal activity, violence and damage to personal property [5].

The reduction of the problem can only occur if effective strategies to prevent, diagnose and treat alcohol abuse are developed.

With regard to the diagnosis it is necessary to find ways that will highlight early use and consequently cancel or minimize the harm related to alcohol. For years, there have been questionnaires (CAGE, AUDIT or MUST) [6, 7], which are self-completed and as such the responses do not always reflect the true consumption.

Real help could come from laboratory tests that represent objective evidence of alcohol problems.

There are many biochemical markers of alcohol abuse, some direct, others indirect, some traditional and others emerging, of which the sensitivity and specificity are not 
always known, with sometimes contradictory data. Therefore the use of different biological matrices such as blood, urine, hair, meconium, could open new horizons.

It is also important to correctly interpret the results obtained in the light of different application areas - clinical, administrative and forensic- to correctly set each case and ensure either the health and safety of the individual and the community.

\section{SENSIBILITY, SPECIFICITY, POSITIVE AND NEGA- TIVE PREDICTIVE VALUES OF BIOMARKERS}

A biochemical marker is a substance present in biological fluids, able to detect the presence or progression of a disease. An ideal biomarker for alcohol abuse should give reliable and reproducible results, be able to differentiate between the different amounts of alcohol consumed, even over time, be easy to use, repeatable, quickly available in all laboratories and economic.

For our purposes it must be sensitive, i.e. able to correctly identify people who have consumed any alcohol, and specific, i.e. able to correctly identify those who have not consumed any alcohol. Furthermore, the ideal biomarker should be measurable for a sufficiently long time even after taking small amounts of alcohol.

If very sensitive it should not give false negatives, if very specific it should not give false positive results. So, in theory, the ideal biomarker should be characterized by sensitivity and specificity of $100 \%$, but this is hardly ever achieved. Actually, it is obvious that there is an intra- and interindividual biological variation and one individual or different individuals may metabolize alcohol at different times and different ways.

Because of this variability, "normal" and "drinking" population show distribution curves partially overlap and grey area represented by this overlap is difficult to interpret. Since the enzymatic metabolism of alcohol is involved in different activities, different people who drink the same amount of alcohol can have different biomarker concentrations. Some individuals may be able to drink excessively without displaying abnormal test results (in these subjects sensitivity is low), on the other hand some alcohol markers may yield abnormal values also in people suffering from non-alcohol related problems, or after taking certain kinds of medication (in these subjects specificity is low.

We have to consider another important aspect: reference interval parameters, or better the cut-off between negative and positive results. If we assume that in the healthy control population (people who drink moderately) the distribution of the measured biomarker is described by a Gaussian curve (this hypothesis is not correct in the majority of cases) the reference interval is calculated as the mean \pm 2 times the standard deviation (SD) of the results obtained. This leads to a specificity lower than $100 \%$ since $5 \%$ of control values are positioned outside of the lowest and the highest limit of reference.

When defining the threshold of positivity for the markers of alcohol abuse it should be considered that the limitation of these studies is that sensitivity and specificity are assessed in relation to self-completed questionnaire as if this were the gold standard for alcohol consumption. In fact, many patients do not actually report accurately their true intake and this creates a validity problem.

Furthermore, the pattern of alcohol consumption and drinking habits vary across cultures and societies, and this causes a variation in the way in which the data is recruited into the healthy control group.

The use of receiver operating characteristics curves (ROC), which evaluates the sensitivity and specificity at different cut-off in the normal and drinking population, has become a tool used to compare the diagnostic performance of biomarkers and selecting optimal thresholds limits.

The likelihood of obtaining a correct classification (predictive value) by the use of a biological marker for alcohol intake is strongly dependant on the prevalence of the use/abuse of alcohol in the population to be studied. The positive predictive value (PPV) gives the proportion of truly positive test results out of all positive results (the sum of true and false positives) while the negative predictive value (NPV) is the proportion of truly negative test results among all negative results (the sum of true and false negatives). When using a marker with fairly high sensitivity and specificity, the risk of misclassification might be rather high if the impairment occurs only infrequently in the study population. Consequently a marker used to identify excessive alcohol consumption perform better (higher PPV) in studies on selected, high risk populations, such as drunk drivers rather than on the general population.

Sensitivity, specificity and predictive values are influenced by the cut off, that is the decision threshold chosen to distinguish between a normal and an abnormal value. It is possible to increase the sensitivity of the test by varying the reference interval, but the specificity becomes reduced. The same is true if you want to increase specificity: it will lose sensitivity.

For general screening, to detect the level of potential hazard for an individual or to evaluate early whether there has been a relapse during rehabilitation the marker to be used must be very sensitive. Conversely, if a marker is to be used to provide evidence in the legal courts, with possible sanctions, such as a loss of employment or revocation of a driving licence, it must be very specific to avoid the possible occurrence of false positives.

\section{EMERGING BIOMARKERS OF ALCOHOL CONSUMPTION}

Recovering ethyl alcohol in biological fluids or in exhaled air is the most traditional and objective evidence to be adopted, however, the measurement of alcohol, due to its fast metabolism, is limited by the time elapsed between intake and determination (poor diagnostic sensitivity).

Also finding a considerable amount of alcohol in the blood of a subject is not indicative of drinking habits nor drink-related social and health problems.

On the other hand, an increase in enzyme activities of aspartato-amino transferase (AST), alanino- amino transferase (ALT), gamma-glutamyl transferase (GGT) or mean corpuscular volume (MCV)) suggests the presence of 
liver damage, not only alcohol-related, but whenever the disease is already advanced (poor diagnostic specificity).

For this reason scientific literature suggests the use of markers more specifically related to the use/abuse of alcohol that can be measured in the body even a long time after ingestion. Furthermore, the increasingly sophisticated analytical technology has allowed to detect also small amounts of these analytes and now it is possible to use alternative biological specimens such as hair and meconium.

\section{Carbohydrate Deficient Transferrin (CDT)}

Transferrin is a glycoprotein which carries and transports iron in the blood. From the structural point of view it possesses from 0 to 2 branched chains of carbohydrates, each of which may end with a number of different sialic acid residues [3]. The most common glycoform contains 4 sialic acid residues and represents the $80 \%$ of the total transferrin.

Alcohol consumption inhibits transferrin sialidation, molecules with two sialic acid residues or without sialic acid residues increase in case of heavy alcohol consumption [3].

The set of glycoforms, poor in sialic acid, known as carbohydrate-deficient transferrin (CDT), is an excellent biomarker for identifying heavy drinkers. CDT has been studied regularly for more than 30 years and has been consolidated the evidence of its performance in the field of alcologics, unlike other very promising markers but explored in a limited way only recently.

As regards the dosage of CDT over the years different methods and techniques have been proposed.

Today the candidate reference method carries out the separation of the glycoforms by HPLC and the detection of iron-transferrin complex at $470 \mathrm{~nm}$, after saturation of the serum sample with an iron salt [8]. The separation of the glycoforms can be carried out also by capillary electrophoresis (CZE) that uses a less specific wavelength $(210 \mathrm{~nm})$ at which many other molecules with similar isoelectric point absorb [9].

These methods are difficult to apply in the clinical laboratory routine where the number of samples to be analyzed is considerable. The need of simplicity and speed has pushed the diagnostic market to develop simplified HPLC and CZE methods included dedicated instrumentation and kits with ready-to-use reagents that permit high workloads and short time runs. On the diagnostic market there is also a direct immunoassay which does not require any pretreatment of the sample [10].

The presence of different methods for the measurement of CDT has been a limit to the use of this biomarker. In fact, different methods give different results for the same sample, hence the need for different thresholds method-depend. Cutoffs range over 1.3 to $1.6 \%$ (commercial CZE), 1.8 to $2.0 \%$ (HPLC) and 2.5\% (immunoassay method). To overcome this difficulty, an international working group was set up to standardize the measurement of CDT, which will aim, amongst other things, to validate the reference material that will allow the alignment of all methods in order to achieve an important objective: getting the same result for the same sample in any laboratory in the world [11].
This is important for all laboratory tests, but especially for those analytes whose results may lead to measures restricting personal freedom. For this reason, when CDT was performed for administrative and legal purposes (driving or firearms license granting, child custody, etc.) is absolutely necessary that the "non-negative" results obtained with screening method are confirmed with a high level analytical techniques (HPLC and CZE), typical of several specialized laboratories where it is possible to send samples for confirmatory analysis.

The half-life of CDT is approximately 14 days and, after 2-3 weeks of abstinence, it usually returns to within normal range.

CDT is increased in people who regularly drink large amounts of alcohol, an daily average intake of at least 40 grams of ethanol (two cans of beer, half a bottle of wine or $120 \mathrm{ml}$ of spirits) for a week is enough to increase the CDT concentration above the cut-off.

The CDT is a very specific marker, unlike in liver enzymes (eg GGT), its increase is only related to the abuse of alcohol, as measured by appropriate methods.

Congenital disorders of glycosylation (CDG), which are diagnosed early in life because they are normally associated with mental retardation, may represent the only circumstances within humans in which CDT measurement is high even without the use of alcohol.

There are also situations where the determination of CDT is complex, as in the case of genetic variants $(<1 \%$ in the population) and in some rare situation of liver disease. This difficulty is linked to the analytical aspect since in these cases it is not possible to perfectly separate the disialotransferrin, the target molecule of CDT, from other transferrin glycoforms. The HPLC is always able to separate all of the glycoforms, including most of the variants, while commercial CZE are not always able to perform a optimal separation. The laboratory must know the limits of own chosen method and as such provide a clear report that will not cause doubts for those who must face and solve any clinical and legal questions.

If the CDT is properly expressed as a percentage of total transferrin, there is no statistically significant difference of concentration between men and women.

In case of pregnancy a gradual increase in the concentration of disialotransferrin has been observed with the progress of gestation, in some cases, at the end of the third quarter of pregnancy, values just above the threshold of positivity can be observed [12]. This is due to the fact that probably the glycoform with a lower content of sialic acid are more saturated with iron, useful for the synthesis of hemoglobin which carries oxygen to the foetus. The distribution of disialotransferrin and other glycoforms with the highest content of sialic acid has been also studied in children and adolescents [13].

When CDT is measured by immunoassay, to improve its performance, an algorithm that takes into account GGT, known as gamma-CDT, has been introduced [14]. This algorithm can sometimes be a confounding factor, especially in traffic medicine [15]. In this case it is better detect CDT by HPLC. 


\section{Etilglucuronide (EtG) and Ethylsulfate (EtS)}

They are direct metabolites from the non-oxidative metabolism of alcohol and represent approximately $0.1 \%$ of alcohol drunk. They are detectable in the urine within one hour of intake, the glucuronide shows an amount significantly higher than the sulfate [16]. Both the metabolites are excreted less quickly than ethanol and therefore the time of detection is a function of the amount of alcohol consumed [17], they are measurable even when the alcohol is no longer detectable. A very sophisticated technique like liquid chromatography coupled to mass spectrometry (LC-MS or LC-MS/MS) is used for their dosage and it allows their simultaneous quantification. Recently an immunoassay method has been introduced into the market that allows the quantification of EtG in urine on the most common analytical platforms in use in clinical laboratories [18].

Urinary EtG and EtS are specific and sensitive markers for detecting the consumption of alcohol even when ethanol can no longer be measured, because for its typical kinetics it is eliminated after a few hours.

The urinary dosage of these markers has an important application in assessing the sobriety of people that are committed to abstinence from alcoholic beverages in a rehabilitation program. EtG and EtS are detectable by a few hours to 2-3 days after intake of small amounts of alcohol such as contained in a light beer [18]. They are also characterised by a large biological variability, it is possible that, after an equal amounts intake of alcohol, people with the same blood alcohol have different levels of EtG and EtS in their urine.

For this reason, it is difficult to estimate the amount of ethanol taken and when the ingestion occurred if it is known only one EtG and EtS measurement. To avoid having an interference from no alcohol-free mouthwashes or disinfectants, it is usually considered as positive threshold a amount greater than $0.5 \mathrm{mg} / \mathrm{L}$ for the $\mathrm{EtG}$, and $0.1 \mathrm{mg} / \mathrm{L}$ for the EtS. The dilution both in vivo (intake of large amounts of liquid) and in vitro (water added to urine after urination) decrease the concentration of EtG and EtS, to minimize this risk of adulteration it is customary to express the result as the ratio between EtG or EtS and urinary creatinine, as is done for the determination of drug abuse in urine [19].

Swedish studies have shown that, in urinary infections, bacterial hydrolysis, which can occur in urine samples contaminated with E. Coli, may give false negative results of EtG [19]. Conversely, in patients with diabetes, fermentation of glucose to ethanol can occur resulting a formation in vitro of EtG, which inevitably leads to false positive results. This does not occur for the EtS [19].

EtG and EtS can also be measured in blood, but these analytes are present for a shorter time than in urine.

The EtG (and not the EtS because of the many disulfide bridges present in the keratin matrix ) is also measured in hair with some success especially in a forensic context despite the problems, still unresolved, related to the use of cosmetics [20].

We do not recommend the only use of EtG in hair to highlight alcohol abuse, because there are no established population-based studies that unequivocally demonstrate the extension of variability between individuals [20, 21] and therefore there could be a high risk of litigation [22]. When interpreting the values of EtG in hair, the Society of Hair Testing (SoTH) has produced a consensus document [23] which proposes the value of $30 \mathrm{pg} / \mathrm{mg}$ scalp hair in the 0-3 up $0-6 \mathrm{~cm}$ proximal segment to be used as a cut-off for alcohol abuse. A threshold of $4 \mathrm{pg} / \mathrm{mg}$ of hair could discriminate the abstainer from moderate drinking. Analysis of samples less than $3 \mathrm{~cm}$ must be avoided or interpreted with caution.

\section{Fatty Acid Ethyl Esters (FAEE)}

Ethyl esters of fatty acids (FAEE), the most important of which are ethyl-stearate, ethyl-palmitate, ethyl-oleate and ethyl-miristate, are produced by the non-oxidative metabolism of alcohol with fatty acids derived from catabolism of triglycerides [24, 25], by of FAEE-synthetase or by acyl-coenzime A: ethanol-O-acyltransferase [25], through a reaction not yet understood in detail.

FAEE are formed in the duodenum and in the bloodstream in the presence of ethanol. In the blood they are carried by albumin or lipoproteins and can be transferred between the different lipid compartments. The albumin causes a rapid secretion of FAEE from the hepatoblastoma cells $\mathrm{HpG} 2$ in culture, while the lipoproteins activate both the synthesis and liberation of FAEE [26].

Their measurement requires very complex instrumentation (GC-MS or LC-MS) [27] and considerable experience in chromatography, knowledge present only in specialized laboratories.

They are measurable in blood, hair, and in various organs such as liver, pancreas, heart and brain, and this suggested the hypothesis that they may play a direct role in the toxic effect of alcohol on organs [28-33]. The elimination curve is similar to that of ethanol, with more prolonged clearance times. Blood levels of FAEE were detectable in serum even 24 hours after alcohol intake [34] and in case of chronic abuse even after 4 days. They have also been suggested to identify binge drinking, a typical heavy episodic drinking where people drink alcoholic beverages with the primary intention of becoming intoxicated by heavy consumption of alcohol over a short period of time [35].

A limitation on their use may be represented by the fact that it is not yet known how much alcohol and what drinking habit are necessary for their formation.

In the diagnosis of chronic alcohol abuse, as well as the monitoring of abstinence, FAEE are promising markers when they are detected in hair, in fact, the ROC curves are able to distinguish between teetotallers, social drinkers and heavy drinkers from alcoholics [29, 36, 37].

They can also be determined after two months of abstinence [38], for which this practice could be useful in monitoring pregnant women [39] and possible perpetrators of crimes under the influence of alcohol [40].

The use of hair care products influence the dosage of FAEE and give false positive results, while incorporation does not depend on the hair pigment.

SoTH has proposed a threshold of $0.5 \mathrm{ng} / \mathrm{mg}$ scalp hair in the $0-3$ up $0-6 \mathrm{~cm}$ proximal segment as the sum of the 
following four esters: ethyl-myristate, ethyl-palmitate, ethyloleate and ethyl-stearate (sensitivity $90 \%$, specificity $90 \%$ ) [23], even if at least fifteen different FAEE have been isolated in hair. When samples less than $3 \mathrm{~cm}$ are used, the results should be interpreted with caution.

The determination of FAEE was recently tried even in the meconium in order to monitor possible foetal exposure to alcohol [40, 41].

\section{Phosphatidylethanol (PEth)}

This name is a generic term for a group of phospholipids formed from phosphatidylcholine (PC) when ethanol is present [42]. Normally PC is transformed into different phosphatidic acids by the enzyme phospholipase $\mathrm{D}$, but the presence of ethanol changes cell membranes and metabolism lead to the formation of PEth which can be used as a specific marker of alcohol use [43].

From a chemical point of view the PEth molecule is constituted by a group of phosphatidylethanol where two long-chain fatty acids are attached. Because they can be different, a lot of different PEth can be present. In human blood the most important molecular species are PEth 16:0/18:1, which contains a residue of oleic acid (16 carbon atoms) and one of palmitic acid (18 carbon atoms) ( the number 0 and the number 1 respectively indicate that the oleic acid has no double bonds in its chain, whereas palmitic acid has one) and 16:0/18:2, but the profile of PEth varies between individuals and may vary over time within the same individual during a relapse [44].

The first technique used for the measurement of PEth was HPLC with evaporative light scattering detector (ESLD) and the total PEth was measured [44, 45].

Recently some methods have been developed in LC-MS and LC-MS/MS in order to quantify different molecular species of PEth in whole blood after extraction from cell membranes [46, 47].

Analysis and instrumentation are quite complex, so it is not easy to perform in standard laboratories, although some simplifications have been proposed [48].

The group of PEth includes many different molecular species which probably vary depending on dietary factors. It has been demonstrated an overall good correlation for PEth 16:0/18:1 and PEth 16:0/18:2 with the total amount. For use as alcohol biomarkers PEth 16:0/18:1 was indicated to be the single most sensitive form of the two, because it usually occurred in highest concentration.

However, the correlation with total PEth was even more precise for the sum of both major forms, which is probably related to the observation that the ratio between PEth16:0/18:1 and 16:0/18:2 differs considerably between samples [46, 47].

The half-life of PEth is about 4 days in the blood and reflects the consumption in the previous weekend. Its concentration is dose-dependent with a big variability between individuals [46], occasional intake of alcohol does not lead to measurable concentrations of PEth by HPLC, while a Swedish study showed that using LC-MS, a very sensitive analytical technique, one third of the donors has a PEth greater than cut-off [48].
Based on these results, in order to indicate the consumption of alcohol, it was calculated as a cut-off 0.2 $\mu \mathrm{mol} / \mathrm{L}$ for PEth $16: 0 / 18: 1$ and $0.7 \mu \mathrm{mol} / \mathrm{L}$ for PEth total respectively [46].

Since the phospholipase $\mathrm{D}$ is also active in vitro, we can not exclude the neoformation of PEth after extraction of the sample in subjects who drink a lot, because ethanol is present inside the blood. For this reason, samples should be frozen immediately at $-20{ }^{\circ} \mathrm{C}$, at this temperature the phospholipase $\mathrm{D}$ is inactive.

If the sample does not contain ethanol, formation in vitro of PEth will not observable even when stored at room temperature for a few days [46].

\section{CONCLUSION}

To prevent the excessive use of alcohol, which can develop into abuse and dependence with negative personal and social effects, it is important to have objective tools that allow early diagnosis.

There are sensitive and specific biochemical markers that can provide quantitative information on the level of alcohol consumption (CDT, ETG, PEth, FAEE etc).

The use of different matrices and the sampling interval can be useful tools for formulating the correct diagnosis.

CDT is currently the most widely used marker of alcohol abuse and one of the most studied; in scientific literature, we can be found a large number of scientific papers describing in detail its behavior, even in relation to some important diseases. At the moment, CDT it is the most specific biomarker to distinguish if a patient drinks regularly a lot.

EtG and EtS in urine find their best application in evaluating the possible use of alcohol in the two/three days prior to urination. It is very useful in monitoring patients needing to follow a rehabilitation program. EtS in urine is not affected by bacterial contamination and by interference due to the presence of glycosuria.

Markers such as FAEE and PEth, whose dosage is still limited to a few research laboratories, need further validation in the epidemiological field, even if PEth in blood appears to be a promising marker.

The use of FAEE is recommended in hair, it is good to keep in mind the recommendations of SoTH for appropriate diagnosis.

To highlight alcohol abuse in the forensic field, such as issuing driver's licenses in subjects who are at risk, blood CDT is used together with scalp hair EtG, whilst the exclusive use of EtG is not recommended.

We can therefore conclude that the use of multiple markers associated with an appropriate medical history are indispensable for the interpretation of individual cases and for the correct formulation of the diagnosis in different aspects of alcohol abuse.

\section{CONFLICT OF INTEREST}

The authors confirm that this article content has no conflict of interest. 


\section{ACKNOWLEDGEMENTS}

Declared none.

\section{REFERENCES}

[1] Room R, Babor T, Rehm J. Alcohol and public health. Lancet 2005; 365: 519-30.

[2] Bianchi V, Raspagni A, Arfini A. Vecchi e nuovi marcatori di abuso alcolico nelle matrici biologiche. 1rd ed. Turin: Ananke 2008 [Italian text : Old and new markers of alcohol abuse in biological matrices].

[3] Bianchi V. Transferrina carboidrato-carente: nuovo marcatore di abuso alcolico. 1rd ed. Turin: Ananke 2006. [ Article in Italian: Carbohydrate-deficient transferrin (CDT) a new marker of alcohol abuse]

[4] World Health Organisation. Global status report on alcohol and health. Available from http://www.who.int/substance abuse/publications/global_alcohol_report/msbgsruprofiles.pdf

[5] Leon DA, Mc Cambridge J. Liver cirrhosis mortality rates in Britain from 1950 to 2001: an analysis of routine data. Lancet 2006; 367: 52-6.

[6] Allen JP, Litten RZ. Recommendations in use of biomarkers in alcoholism treatment trials. Alcohol Clin Exp Res 2003; 27: 166770.

[7] Alling C, Chick JD, Anton R, et al. Revealing alcohol abuse: to ask or to test. Alcohol Clin Exp Res 2005; 29:1257-63.

[8] Helander A, Husa A, Jeppsson JO. Improved HPLC method for carbohydrate-deficient transferrin in serum. Clin Chem 2003; 9:1881-90.

[9] Crivellente F, Fracasso G, Valentini R, et al. Improved method for carbohydrate-deficient transferrin determination in human serum by capillary zone electrophoresis. J Chromatogr B 2000; 739: 8193.

[10] Bianchi V, Arfini C, Helander A. Determination of carbohydratedeficient transferrin (CDT) in Italy. Clin Chem Lab Med 2008; 46: 2759-62.

[11] Helander A, Wielders JP, Jeppsson JO, et al. Toward standardization of carbohydrate-deficient transferrin (CDT) measurements: II. Performance of a laboratory network running the HPLC candidate reference measurement procedure and evaluation of a candidate reference material. Clin Chem Lab Med 2000; 48:1585-92.

[12] Bianchi V, Ivaldi A, Raspagni A, et al. Pregnancy and variations of carbohydrate-deficient transferrin levels measured by the candidate reference HPLC method. Alcohol Alcohol 2011; 46: 123-7.

[13] Bianchi V, Ivaldi A, Raspagni A, et al. High-performance liquid chromatography evaluation of serum carbohydrate-deficient transferrin and more sialylated transferrin glycoforms in children. Scand J Clin Lab Invest 2012; 72: 274-8.

[14] Niemelä O. Biomarker in alcoholism. Clin Chim Acta 2007; 377: 239-49.

[15] Bianchi V, Ivaldi A, Raspagni A, et al. Carbohydrate-deficient transferrin (CDT) alone and combinated with GGT $(\gamma$-CDT) in traffic medicine Alcohol Alcohol 2010; 3: 306-11

[16] Helander A, Beck O. Mass spectrometric identification of ethyl sulphate as an ethanol metabolite in humans. Clin Chem 2004; 50: 936-7.

[17] Helander A, Böttcher M, Fehr C, et al. Detection times for urinary ethyl glucuronide and ethyl sulfate in heavy drinkers during alcohol detoxification. Alcohol Alcohol 2009: 44: 55-61.

[18] Bianchi V, Piccinini S, Raspagni A, et al. Test immunoenzimatico per il dosaggio in fase omogenea per il dosaggio dell'etilglucuronide nelle urina. [Omogeneous phase immunoassay for ethyl glucuronide measurement]. Minerva Med Leg 2009; 129: 177-82.

[19] Helander A, Olsson I, Dahl H. Postcollection syntesis of ethyl glucuronide by bacteria in urine may cause false identification of alcohol consumption. Clin Chem 2007; 53: 1-3.

[20] Martins Ferreira L, Binz T, Yegles M. The influence of ethanol containing cosmetics on ethyl glucuronide concentration in hair. Forensic Sci Int 2012; 218: 123-5

[21] Tagliaro F, Bortolotti F, Viel G, et al. Caveats against an improper use of hair testing to support the diagnosis of chronic excessive alcohol consumption, following the "Consensus" of the Society of Hair Testing 2009. Forensic Sci Int 2010; 207: e69-e70.
[22] Pragst F. Interpretation problems in a forensic case of abstinence determination using alcohol markers in hair. Forensic Sci Int 2012; 217: e4-7.

[23] Society of Hair Testing (SoTH). Consensus of the Society of Hair Testing on hair testing for chronic excessive alcohol consumption 2011. Available at: www.soth.org

[24] Hannuksela ML, Liisanantii MK, Savolainen MJ. Effect of alcohol on lipids and lipoproteins in relation to atherosclerosis. Crit Rev Clin Lab Sci 2003; 39: 225-83.

[25] Diczfalusy MA, Bjorkhem I, Einarsson C, et al. Characterization of enzymes involved in formation of ethyl esters of long-chain fatty acids in humans. J Lipid Res 2001; 42: 1025-32.

[26] Hasaba A, LaposataM. The synthesis and secretion of fatty acid ethyl esthers from HepG2 cells are stimulated by lipoproteins and albumin. Alcohol Clin Exp Res 2001; 25: 338-43.

[27] Pragst F, Auwaerter V, Sporkert F, et al. Analysis of fatty acid ethyl esthers of long chain in hair as possible markers of chronically elevated alcohol consumption by headspace solid-phase microextraction (HS-SPME) and gas chromatography-mass spectrometry (GC-MS). Forensic Sci Int 2001; 121: 76-88.

[28] Bird DA, Laposata M, Hamilton JA. Binding of ethyl oleate to low density lipoprotein, phospholipid vesicles and albumin: a $13 \mathrm{C}$ NMR study. J Lipid Res 1996; 37: 1449-58.

[29] Laposata EA, Lange LG. Presence of nonoxidative ethanol metabolism in human organs commonly damaged by ethanol abuse. Science 1986; 31:497-9.

[30] Laposata M. Fatty Acid ethyl esters: ethanol metabolites which mediate ethanol-induced organ damage and serve as markers of ethanol intake. Prog Lipid Res 1998; 37: 307-16.

[31] Vonlaufen A, Wilson JS, Pirola RC, et al. Role of alcohol metabolism in chronic pancreatitis, Alcohol Res Health 2007; 30: 48-54.

[32] Doyle KM, Cluette-Brown JE, Dube DM, et al. Fatty Acid ethyl esters in the blood as markers for ethanol intake. JAMA 1996; 276: 1152-6.

[33] Borucki K, Dierkers J, Warteberg J, et al. In heavy drinkers, fatty acid ethyl esters remain elevated for up to 99 hours. Alcohol Clin Exp Res 2007; 31: 423-7.

[34] Soderberg BL, Salem RO, Best CA. Fatty acid ethyl esters. Ethanol metabolites that reflect ethanol intake. Am J Clin Pathol 2003; 119: S94-9.

[35] Wurst FM, Alexson S, Wolfersdorf M, et al. Concentration of fatty ethyl esters in hair of alcoholics: comparison to other biological state markers and self reported ethanol intake. Alcohol Alcohol 2004; 39: 33-8

[36] Yegles M, Labarthe M, Auwarter, et al. Comparison of ethylglucuronide and fatty acid ethyl esters concentrations in hair of alcoholics, social drinkers and teetotalers. Forensic Sci Int 2004; 145: 167-73.

[37] Wurst FM, Kelso E, Weinmann W, et al. Measurement of direct ethanol metabolites suggests higher rate of alcohol use among pregnant women than found with the AUDIT- a pilot study in a population-based sample of Swedish women. Am J Obstet Gynecol 2008; 407: e1-e5.

[38] Wurst FM, Yegles M, Alling C, et al. Measurement of direct ethanol metabolites in a case of a former driving under the influence (DUI) of alcohol offender, now claiming abstinence. Int J Leg Med 2008; 122: 235-9.

[39] Morini L, Marchei E, Pellegrini M, et al. Liquid chromatography with tandem mass spectrometric detection for the measurement of ethyl glucuronide and ethyl sulfate in meconium: new biomarkers of gestational ethanol exposure? Ther Drug Monit 2008; 30: 72532 .

[40] Bakdash A, Burger P, Goecke TW, et al. Quantification of fatty acid ethyl esters (FAEE) and ethyl glucuronide (EtG) in meconium from newborns for detection of alcohol abuse in a maternal health evaluation study. Anal Bioanal Chem 2010; 396: 2469-77.

[41] Gustavvson L, Allig C. Blood phosphatidylethanol in rat brain by phospholipidae D. Biochem Biophys Res Commun 1987; 142: 95863.

[42] Aradottir S, Asanovska G, Gjerss S, et al. Phosphatidylethanol (PEth) concentrations in blood are correlated to reported alcohol intake in alcohol-dependent patients. Alcohol Alcohol 2006; 41: 431-7.

[43] Gunnarsson T, Karlsson A, Hansson P, et al. Determination of phosphatidylethanol in blood from alcoholic males using high- 
performance liquid chromatography and evaporative light scattering or electrospray mass spectrometric detection. J Chromatogr B Biomed Sci Appl 1998; 705: 243-9.

[44] Helander A, Zheng Y. Molecular species of the alcohol biomarker phosphatidylethanol in human blood measured by LC-MS. Clin Chem 2009; 55: 1395-405.

[45] Aradottir S, Olsson BL. Methodologic modifications on quantification of phosphatidylethanol in blood from humans abusing alcohol, using high-performance liquid chromatography and evaporative light scattering. BMC Biochem 2005; 6: 18.
[46] Nalesso A, Viel G, Cecchetto G, et al. Quantitative profiling of phosfatidyl molecular species in human blood by liquid chromatography high resolution mass spectrometry. J Chromatogr A 2011; 1218: 8423-31.

[47] Zheng Y, Beck O, Helander A. Method development for routine liquid chromatography-mass spectrometry measurement of the alcohol biomarker phosphatidylethanol (PEth) in blood. Clin Chim Acta 2011; 412: 1428-35.

[48] Helander A. Biomarkör kan fånga tidigt riskbruk av alcohol. Läkartidningen 2011; 108: 2291-5 [article in Swedish: Biomarkers can early detect hazardous drinking of alcohol].

Received: March 22, 2013

Revised: April 02, 2013

Accepted: April 02, 2013

(C) Bianchi et al.; Licensee Bentham Open.

This is an open access article licensed under the terms of the Creative Commons Attribution Non-Commercial License (http://creativecommons.org/licenses/by$\mathrm{nc} / 3.0 /$ ), which permits unrestricted, non-commercial use, distribution and reproduction in any medium, provided the work is properly cited. 